\title{
Left ventricular remodeling in hypertrophic cardiomyopathy patients with atrial fibrillation
}

Hongwei Tian, Jingang Cui, Chengzhi Yang, Fenghuan Hu, Jiansong Yuan, Shengwen Liu, Weixian Yang, Xiaowei Jiang and Shubin Qiao* (D)

\begin{abstract}
Background: Atrial fibrillation (AF) is the most common complication in hypertrophic cardiomyopathy (HCM). The mechanisms of AF is associated with left atrial (LA) structural remodeling in HCM patients. However, the impact of left ventricular (LV) remodeling on the presence of AF in HCM patients has not been evaluated yet. We sought to investigate effect of LV remodeling on the presence of AF assessed by cardiovascular magnetic resonance (CMR) in HCM patients.

Methods: A total of 394 HCM patients were enrolled into this study, including HOCM patients $(n=293)$ and NOHCM patients $(n=101)$. Patients were divided into HCM with AF $(50)$ and HCM without AF $(n=344)$. Data were collected from hospital records.

Results: LA diameter and LV remodeling index (LVRI) were significantly higher in HCM patients with AF than that of $\mathrm{HCM}$ patients without AF $(46.6 \pm 7.4 \mathrm{~mm}$ versus $39.9 \pm 8.0 \mathrm{~mm}, p<0.001$, and $1.46 \pm 0.6$ versus $1.2 \pm 0.4, p=0.002$, respectively). HCM patients with AF were older than HCM patients without AF (53.6 \pm 11.7 years versus $47.7 \pm 13$. 6 years, $p=0.002)$. Additionally, LVRI positively correlated to LA size $(r=0.12, p=0.02)$. In a multivariable logistic regression analysis, when adjusting for age and LV end diastolic mass index, LVRI and LA size remained an independent determinant of $A F$ in HCM patients $(\mathrm{OR}=4.7, p=0.001$ and $\mathrm{OR}=1.13, P<0.001)$.

Conclusion: HCM patients with AF showed significantly more LA diameter, LVRI and age than HCM patients without AF. LVRI and LA size were strong independent predictor of AF in HCM, suggesting LV remodeling may contribute to the occurrence of AF in HCM patients.
\end{abstract}

Keywords: Atrial fibrillation, Hypertrophic cardiomyopathy, Left ventricular remodeling

\section{Background}

Hypertrophic cardiomyopathy (HCM) is a complex and relatively common form of genetic heart disease characterized by left ventricular (LV) hypertrophy and the most frequent cause of sudden death in the young [1]. Histologically, HCM is characterized by myocyte disarray, scarring and microvascular dysfunction [2].

Atrial fibrillation (AF) is the most common arrhythmia in HCM and was associated with an increased risk for morbidity and mortality [3, 4]. The

\footnotetext{
* Correspondence: qsbfw@@ina.com

State Key Laboratory of Cardiovascular Disease, Fuwai Hospital, National Center for Cardiovascular Diseases, Chinese Academy of Medical Sciences and Peking Union Medical College, Beijing 100037, China
}

mechanisms of AF are complex and associated with structural and electrical remodeling in the atria and ventricular myocardium $[5,6]$. In $\mathrm{HCM}$ patients, increased LA size, late gadolinium-enhancement (LGE) and advanced age have been shown to be independent predictors of the presence of AF [7, 2, 8]. However, the impact of LV remodeling on the presence of AF in HCM patients has not been evaluated yet. Thus, we used cardiovascular magnetic resonance (CMR) to evaluate effect of left ventricular remodeling index (LVRI) on the presence of AF in HCM patients.

(c) The Author(s). 2018 Open Access This article is distributed under the terms of the Creative Commons Attribution 4.0 International License (http://creativecommons.org/licenses/by/4.0/), which permits unrestricted use, distribution, and reproduction in any medium, provided you give appropriate credit to the original author(s) and the source, provide a link to the Creative Commons license, and indicate if changes were made. The Creative Commons Public Domain Dedication waiver (http://creativecommons.org/publicdomain/zero/1.0/) applies to the data made available in this article, unless otherwise stated. 


\section{Methods}

\section{Study population}

The protocol study was approved by Fuwai Hospital ethics committee. The informed consents were obtained from all participants. We retrospectively analyzed data from 440 HCM patients who had maximum LV wall thickness $\geq$ $15 \mathrm{~mm}$ (or $\geq 13 \mathrm{~mm}$ with an unequivocal family history of HCM) in the absence of other cardiac or systemic causes of left ventricular hypertrophy $[9,10]$ between November 2012 and August 2016. Evaluation of patients included complete medical history, blood examination, physical examination, 24-h ambulatory electrocardiographic monitoring, transthoracic echocardiography, invasive coronary angiography, 12-lead electrocardiography and cardiovascular magnetic resonance imaging (MRI). Patients were excluded if they had (1) coronary artery disease (coronary artery stenosis $>50 \%$ ), (2) renal dysfunction, (3) heart failure, (4) cardiac valve disease, (5) permanent mechanical device implantation. Forty-six patients were excluded owing to concomitant coronary artery disease $(n=44)$ and cardiac valve disease $(n=2)$ (Fig. 1). Finally, a total of 394 patients were enrolled into this study, including HOCM patients $(n=293)$ and NOHCM patients $(n=101)$. Patients were divided into HCM with AF (50) and HCM without AF $(n=344)$.

\section{Echocardiography}

Standard transthoracic M-mode, 2-dimensional, and pulse-wave and continuous-wave Doppler images were obtained with an iE33 Color Doppler Ultrasound System (Philips Healthcare, Andover, Massachusetts). All measurements were analyzed following the guidelines of the American Society of Echocardiography. The left ventricular outflow tract (LVOT) peak gradient was estimated using the simplified Bernoulli eq. HCM with obstruction was defined as an LVOT peak gradient $\geq 30 \mathrm{mmHg}$ at rest or provoked LVOT peak gradient > $50 \mathrm{mmHg}$. Patients were divided into non-obstructive (NOHCM) or obstructive (HOCM) based on left ventricular outflow tract obstruction [11].

\section{Cardiovascular MRI}

CMR was performed using a 1.5-T speed clinical scanner (Magnetom Avanto; Siemens Medical Solutions, Erlangen, Germany). All MR image was analysed by a single experienced observer who was blinded to the all HCM patients. Endocardial contours of the LV myocardium were manually traced at end-diastole and end-systole on each LV short-axis cine image. LV end-diastolic volume (LVEDV), stroke volume, LV end-systolic volume (LVESV), LV

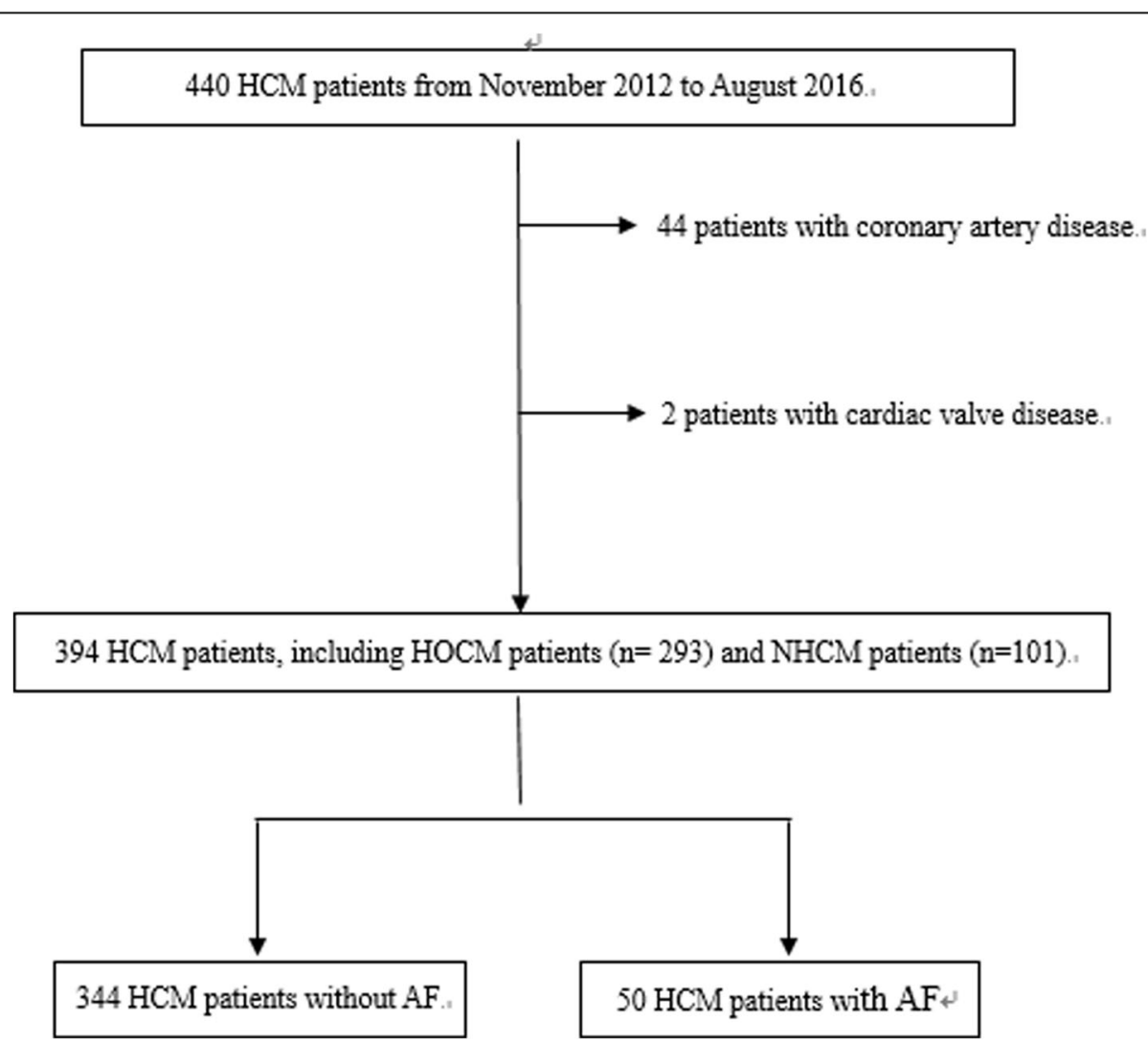

Fig. 1 Flow chart of patient inclusion in the current study. LA, left atrial; HCM, hypertrophic cardiomyopathy; NOHCM, non-obstructive hypertrophic cardiomyopathy; HOCM, obstructive hypertrophic cardiomyopathy 

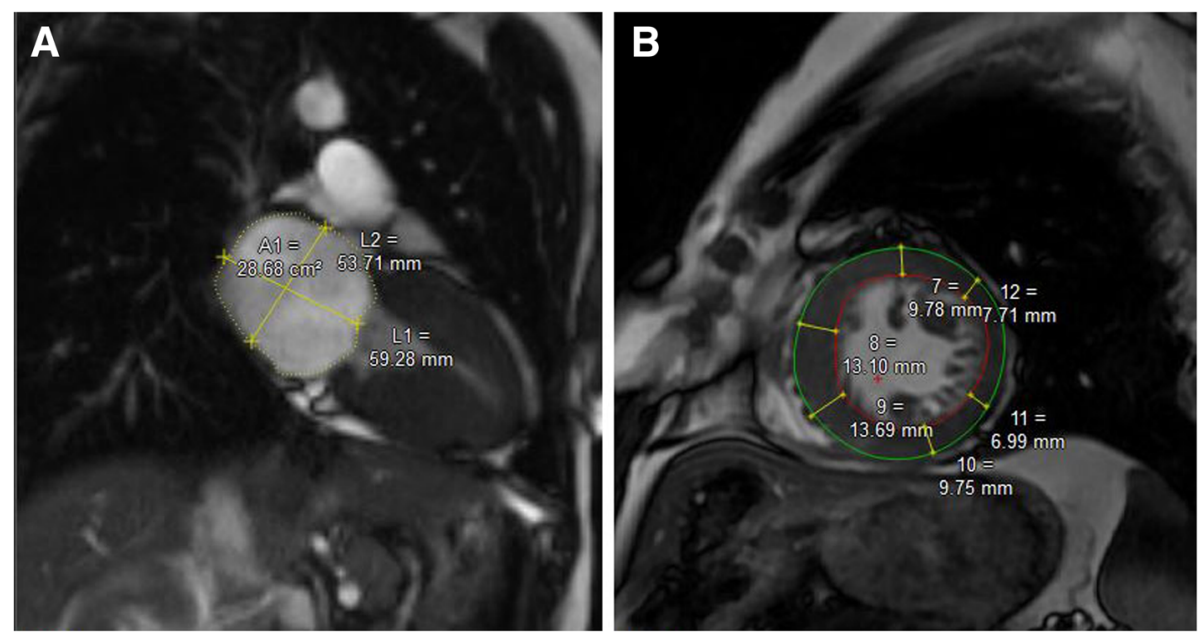

Fig. 2 CMR images showed LA size (a) (yellow line) and contours of endocardial and epicardial (b) (red circle and green circle). CMR, cardiovascular magnetic resonance; LA, left atrial

ejection fraction $(\mathrm{EF})$, and cardiac output were then calculated in a standard fashion. The LV end-diastolic diameter (EDD) was measured from short axis at LV end-diastolic phase and left atrial diameter (LAD) (Fig. 2a) was measured from transverse axis at LV end-systolic phase [11]. Left ventricular mass (LVM) was obtained on the basis of end-diastolic endocardial and epicardial contours (Fig. 2b) and calculated as the product of myocardial volume and specific density of myocardial tissue $(1.05 \mathrm{~g} / \mathrm{ml})$. LVM and
LV EDV were indexed to body surface area. Left ventricular remodeling index $(\mathrm{LVRI}=\mathrm{LVM} / \mathrm{LV}$ EDV) was calculated used the methods described previously [12].

\section{Atrial fibrillation}

The diagnosis of AF was based on 12-lead electrocardiography or $24 \mathrm{~h}$ dynamic electrocardiogram recordings, or by an established history of paroxysmal or chronic AF [13].

Table 1 Patient Demographics and Baseline Characteristics

\begin{tabular}{|c|c|c|c|c|}
\hline Variable & All Patients $(n=394)$ & Patients with AF $(n=50)$ & Patients without AF $(n=344)$ & $P$ value \\
\hline Age, y & $48.5 \pm 13.5$ & $53.6 \pm 11.7$ & $47.7 \pm 13.6$ & 0.002 \\
\hline Male, n (\%) & $247(62.7 \%)$ & 37 (74\%) & $210(61 \%)$ & 0.08 \\
\hline Body surface area, $\mathrm{m}^{2}$ & $1.8 \pm 0.2$ & $1.8 \pm 0.3$ & $1.8 \pm 0.2$ & 0.41 \\
\hline NYHA class & $2.4 \pm 0.9$ & $2.5 \pm 0.9$ & $2.4 \pm 0.9$ & 0.45 \\
\hline Heart rate, beats/min & $70.5 \pm 10.3$ & $68.6 \pm 9.9$ & $70.8 \pm 10.4$ & 0.15 \\
\hline SBP (mmHg) & $118.7 \pm 17.2$ & $120.0 \pm 19.0$ & $118.8 \pm 15.7$ & 0.63 \\
\hline $\mathrm{DBP}(\mathrm{mmHg})$ & $73.0 \pm 10.3$ & $74.5 \pm 10.9$ & $72.7 \pm 9.9$ & 0.28 \\
\hline Syncope, n (\%) & $97(24.6 \%)$ & $13(26 \%)$ & $84(24.4 \%)$ & 0.81 \\
\hline Dyspnea, n (\%) & 319 (81\%) & $42(84 \%)$ & $277(80.5 \%)$ & 0.56 \\
\hline Hypertension, n (\%) & $116(29.4 \%)$ & $18(36 \%)$ & $98(28.5 \%)$ & 0.28 \\
\hline Diabetes mellitus, n (\%) & $13(3.3 \%)$ & $4(8 \%)$ & $9(2.6 \%)$ & 0.05 \\
\hline Family history of HCM, n (\%) & $56(14.2 \%)$ & $7(14 \%)$ & 49 (14.2\%) & 0.96 \\
\hline Family history of SCD, n (\%) & $24(6.1 \%)$ & $1(2 \%)$ & $23(6.7 \%)$ & 0.33 \\
\hline \multicolumn{5}{|l|}{ Medications, n (\%) } \\
\hline$\beta$-Blockers & $261(66.2 \%)$ & $36(72 \%)$ & $225(65.4 \%)$ & 0.36 \\
\hline \multicolumn{5}{|l|}{ Echocardiography } \\
\hline Systolic anterior motion & $293(74.4 \%)$ & $36(72 \%)$ & $257(74.7 \%)$ & 0.68 \\
\hline LVOTPG at rest (mmHg) & $74.9 \pm 37.1$ & $68.4 \pm 46.9$ & $75.9 \pm 35.4$ & 0.34 \\
\hline
\end{tabular}

HCM hypertrophic cardiomyopathy, LV left ventricular, LVOTG LV outflow tract gradient, NS not significance; Values are expressed as either mean \pm SD or number (percentage) 
Table 2 CMR assessment

\begin{tabular}{llll}
\hline Variable & Patients with AF & Patients without AF & $P$ value \\
\hline LA dimension, $\mathrm{mm}$ & $46.6 \pm 7.4$ & $39.9 \pm 8.0$ & $<0.001$ \\
LVEDD, mm & $47.0 \pm 5.1$ & $46.5 \pm 5.3$ & 0.48 \\
LV ejection fraction, \% & $63.4 \pm 12.3$ & $67.2 \pm 9.8$ & 0.015 \\
Septal wall thickness, $\mathrm{mm}$ & $26.6 \pm 4.5$ & $24.7 \pm 5.5$ & 0.26 \\
LV end diastolic volume index, $\mathrm{ml} / \mathrm{m}^{2}$ & $69.6 \pm 21.9$ & $70.7 \pm 16.7$ & 0.72 \\
LV ESVI & $26.3 \pm 14.5$ & $23.7 \pm 11.4$ & 0.22 \\
Cl & $3.0 \pm 1.0$ & $3.2 \pm 0.8$ & 0.14 \\
LV end diastolic mass index, $\mathrm{g} / \mathrm{m}^{2}$ & $95.8 \pm 36.7$ & $85.7 \pm 34.8$ & 0.07 \\
LVRI & $1.46 \pm 0.6$ & $1.2 \pm 0.4$ & 0.002 \\
\hline
\end{tabular}

Data are presented as \pm standard deviation. Volumes are indexed to body surface area. EDD end diastolic dimension, $L A$ left atrial, $L V$ left ventricular, ESVI endsystolic volume index, $\mathrm{Cl}$ Cardiac index

\section{Statistical analysis}

Statistical calculations were performed using SPSS 20.0 (SPSS Inc.; Chicago, IL, USA). In the case of a $p<0.05$, the result was considered statistically significant. Data are expressed as mean \pm SD for normally distributed continuous variables. Differences between means were measured by Student's t-tests. Noncontinuous data were compared by chi-square tests as appropriate. Pearson correlation was used to evaluate the correlation between LA size and LVRI. Multivariate analysis was performed with logistic regression analysis using block entry of the following variables: LVRI, LA size, LV end diastolic mass index, and age to evaluate if these variables were independent predictors of $\mathrm{AF}$, provided to have a $p<0.10$ in univariate analysis.

\section{Results}

\section{Patients characteristics}

In our study, AF was documented in $50 \mathrm{HCM}$ patients (12.7\%). Baseline characteristics are presented in Table 1. No significant differences were observed for gender, systolic blood pressure (SBP), diastolic blood pressure (DBP), heart rate, NYHA class, body surface area (BSA), LVOT peak gradient. HCM patients with AF were older than HCM patients without AF $(53.6 \pm 11.7$ years versus $47.7 \pm 13.6$ years, $p=0.002$ ). The proportion of syncope, dyspnea, family history of HCM, family history of SCD, diabetes mellitus, hypertension, systolic anterior motion and use of medications did not differ significantly between HCM patients with AF and without AF groups.

LA and LV parameters, LV end diastolic mass index and LVRI were all comparable between HCM patients with $\mathrm{AF}$ and without $\mathrm{AF}$, Table 2. Left atrial diameter and LVRI were significantly higher in HCM patients with AF than that of $\mathrm{HCM}$ patients without AF (46.6 \pm $7.4 \mathrm{~mm}$ versus $39.9 \pm 8.0 \mathrm{~mm}, p<0.001$, and $1.46 \pm 0.6$ versus $1.2 \pm 0.4, p=0.002$ ). Additionally, pearson correlation analysis showed LVRI positively correlated to LA size $(r=0.12, p=0.02)$ in all HCM patients, Fig. 3 .
In HOCM patients, LA dimension ( $p=0.025)$, LV ejection fraction $(p<0.001)$, septal wall thickness $(p<0.001)$, LV end diastolic mass index $(p<0.001)$ and LVRI $(p<0.001)$ were significantly larger and LV EDD $(p<0.001)$ was lower compared to NOHCM patients. However, there was no significant differences between HOCM patients and NOHCM patients regarding the occurrence of $\mathrm{AF}(12.6 \%$ vs. $12.9 \%$, $p=0.95$ ), see Table 3 .

In a multivariable logistic regression analysis, when adjusting for age and LV end diastolic mass index, LVRI and LA size remained an independent determinant of $\mathrm{AF}$ in $\mathrm{HCM}$ patients $(\mathrm{OR}=4.7, p=0.001$ and $\mathrm{OR}=1.13$, $P<0.001)$, see Table 4 .

\section{Discussion}

The present study demonstrates that HCM patients with AF had higher LA diameter, age and LVRI than HCM patients without AF. LA size mildly correlated to LVRI in all HCM patients. When adjusting for age and LV end

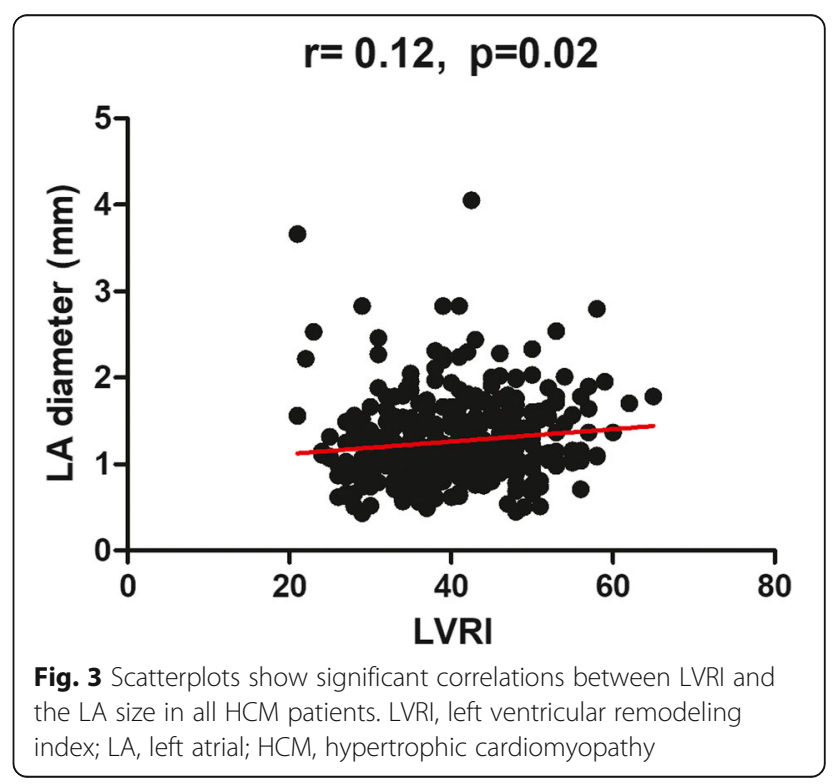


Table 3 Comparison of left ventricular and left atrial dimensions between HOCM and NOHCM patients

\begin{tabular}{llll}
\hline Variable & HOCM & NOHCM & $P$ value \\
\hline AF, \% & $12.6 \%$ & $12.9 \%$ & 0.95 \\
LA dimension, mm & $40.4 \pm 8.5$ & $38.4 \pm 7.7$ & 0.025 \\
LVEDD, mm & $46.0 \pm 5.2$ & $48.3 \pm 5.0$ & $<0.001$ \\
LV ejection fraction, \% & $68.5 \pm 9.2$ & $61.7 \pm 11.2$ & $<0.001$ \\
Septal wall thickness, mm & $23.6 \pm 5.4$ & $21.0 \pm 6.3$ & $<0.001$ \\
LV end diastolic volume index, $\mathrm{ml} / \mathrm{m}^{2}$ & $71.4 \pm 18.6$ & $68.2 \pm 13.2$ & 0.12 \\
LV ESVI, ml/m ${ }^{2}$ & $23.1 \pm 11.7$ & $26.8 \pm 11.9$ & 0.008 \\
Cl, $\mathrm{ml} / \mathrm{m}^{2}$ & $3.3 \pm 0.9$ & $2.8 \pm 0.7$ & $<0.001$ \\
LV end diastolic mass index, $\mathrm{g} / \mathrm{m}^{2}$ & $91.4 \pm 35.5$ & $74.4 \pm 31.0$ & $<0.001$ \\
LVRI & $1.3 \pm 0.5$ & $1.1 \pm 0.5$ & $<0.001$ \\
\hline AF atia fbriltion, LA left atral EDD & dias &
\end{tabular}

$A F$ atrial fibrillation, $L A$ left atrial, $E D D$ end diastolic dimension, $L V$ left ventricular, ESVI end-systolic volume index, CI Cardiac index, LVRI left ventricular remodeling index

diastolic mass index, LVRI and LA size remained an independent determinant of AF in HCM patients.

$\mathrm{AF}$ is a commonly reported complication in HCM that affects quality of life and increases risk for morbidity and mortality. It has been previously revealed that the diagnosis of HCM precedes the presence of AF in the majority of HCM patients [3] which strongly suggests that the structural and physiological changes related to the development of AF. In HCM patients, diastolic dysfunction, advanced age, myocardial ischemia, myocardial fibrosis, LA diameter and congestive heart failure symptoms have been shown to be associated with the development of AF [7, 14]. However, the impact of LV remodeling on the presence of AF in HCM patients has not been evaluated yet. The aim of the present study was to investigate whether LV remodeling is related to the occurrence of AF in HCM patients.

LA dimension is one of the most important determinants of AF occurrence in HCM patients. In our study, we showed that LA diameter and age was significantly higher in HCM patients with AF than that of HCM patients without AF, these findings confirm previous study $[2,13,15]$. In the present study, we also showed LVRI positively correlated to LA size, suggesting that LV remodeling may contribute to the enlargement of LA. LA enlargement is a multifactorial process in HCM, including LA overload, mitral regurgitation, intrinsic myocardial stiffness, LV diastolic dysfunction and rhythm disturbances $[14,16,17]$.

The LVRI which was calculated as the ratio of LV mass and end-diastolic volume can evaluate the degree of LV remodeling [6]. In our study, HCM patients with AF had higher LV mass index and LVRI. In a multivariable logistic regression analysis, LVRI and LA size remained an independent determinant of AF in HCM patients. These observations indicate that LA size and progressive LV remodeling may contribute to the occurrence of AF in HCM patient. The main underlying structural abnormalities in HCM include myocardial cell disarray, coronary microvasculature dysfunction and remodeling changes [18, 19]. LV myocardial remodeling that occur as a compensatory mechanism and can involve changes to the fibroblasts, myocytes and interstitium. LV remodeling and increased LV mass impaired diastolic function due to increased myocardial stiffness and decreased chamber compliance [17]. Moreover, LV diastolic dysfunction can lead to LA enlargement and associated rhythm disturbances [20]. Patients with AF frequently have the left atrial appendage remodeling in which there is dilation, stretching, and reduction in pectinate muscle volume [21]. Prior studies have showed that LA diameter and $\mathrm{P}$ wave dispersion values are the most significant predictors for AF occurrence in patients with HCM [22]. All these findings suggested that the AF was a result of electrical remodeling and myocardial remodeling [23].

\section{Limitations}

There may be some limitations in our study. Firstly, we did not evaluate the impact of late gadolinium enhanced (LGE) on the presence of AF in HCM patients owning to the absence of LGE examination. Secondly, in this study, patients with hypertension were not excluded.

\section{Conclusions}

HCM patients with AF showed significantly more LA diameter, LVRI and age than HCM patients without AF. LVRI and LA size were strong independent predictor of AF in HCM, suggesting that the LA enlargement and progressive LV remodeling may contribute to the occurrence of $\mathrm{AF}$ in $\mathrm{HCM}$ patients.

Table 4 Predictors of AF in HCM group by univariate and multivariable logistic regression

\begin{tabular}{|c|c|c|c|c|c|c|}
\hline \multirow[t]{2}{*}{ Variable } & \multicolumn{3}{|c|}{ Univariate analysis } & \multicolumn{3}{|c|}{ Multivariable logistic regression } \\
\hline & $P$ value & Crude OR & $95 \% \mathrm{Cl}$ & $P$ value & Adjusted OR & $95 \% \mathrm{Cl}$ \\
\hline Age & 0.004 & 1.0 & $1.0 \sim 1.1$ & 0.01 & 1.0 & $1.0 \sim 1.1$ \\
\hline LA dimension, mm & $<0.001$ & 1.1 & $1.1 \sim 1.2$ & $<0.001$ & 1.13 & $1.1 \sim 1.2$ \\
\hline LV end diastolic mass index, $\mathrm{g} / \mathrm{m}^{2}$ & 0.06 & 1.0 & $1.0 \sim 1.02$ & 0.07 & 0.99 & $0.97 \sim 1.0$ \\
\hline LVRI & 0.003 & 2.3 & $1.3 \sim 4.0$ & 0.001 & 4.7 & $1.9 \sim 11.8$ \\
\hline
\end{tabular}

$L A$ left atrial, $L V$ left ventricular, $L V R I$ left ventricular remodeling index 


\section{Abbreviations}

AF: Atrial fibrillation; CMR: Cardiovascular magnetic resonance; DBP: Diastolic blood pressure; EDD: End-diastolic diameter; EF: Ejection fraction; HCM: Hypertrophic cardiomyopathy; HOCM: Obstructive hypertrophic cardiomyopathy; LA: left atrial; LAD: Left atrial diameter; LGE: Late gadolinium-enhancement; LV: Left ventricular; LVEDV: LV end-diastolic volume; LVESV: LV end-systolic volume; LVM: Left ventricular mass; LVOT: Left ventricular outflow tract; LVRI: LV remodeling index; NOHCM: Nonobstructive hypertrophic cardiomyopathy; SBP: systolic blood pressure

\section{Acknowledgements}

The authors thank the study patients for participating and the study personnel for their invaluable contribution. The authors also thank All staffs at the department of nuclear magnetic resonance for performing and assisting with the cardiovascular MRI analysis.

\section{Funding}

This study was supported by grants from the National Natural Science Foundation of China (nos. 81370327).

\section{Availability of data and materials}

According to the Fuwai Hospital system, we are not allowed to share original study data publicly.

\section{Authors' contributions}

HWT, SBQ, CZY, and JGC contributed to the design of the study. HWT, JSY and FHH contributed to the analysis, while all authors (HWT, JGC, CZY, FHH, JSY, SWL, WXY, XWJ, SBQ) contributed to the interpretation of data. HWT drafted the manuscript and SWL, WXY, and XWJ contributed significantly to the preparation. All the authors critically revised the manuscript and gave final approval and agree to be accountable for all aspects of the work, ensuring both its integrity and accuracy.

\section{Ethics approval and consent to participate}

Approval of the Ethics Committee of Peking Union Medical College Affiliated Fuwai Hospital was obtained before the start of the work, and each participant signed written consent form.

\section{Consent for publication}

Not applicable.

\section{Competing interests}

The authors declare that they have no competing interests.

\section{Publisher's Note}

Springer Nature remains neutral with regard to jurisdictional claims in published maps and institutional affiliations.

Received: 9 August 2018 Accepted: 22 October 2018 Published online: 03 November 2018

\section{References}

1. Maron BJ. Hypertrophic cardiomyopathy: a systematic review. Jama. 2002; 287(10):1308-20.

2. Papavassiliu T, Germans T, Flüchter S, Doesch C, Suriyakamar A, Haghi D, et al. CMR findings in patients with hypertrophic cardiomyopathy and atrial fibrillation. J Cardiovasc Magn Reson. 2009:11(1):1-9.

3. Olivotto I, Cecchi F, Casey SA, Dolara A, Traverse JH, Maron BJ. Impact of atrial fibrillation on the clinical course of hypertrophic cardiomyopathy. Circulation. 2001;104(21):2517-24.

4. Doi $Y$, Kitaoka H. Hypertrophic cardiomyopathy in the elderly: significance of atrial fibrillation. J Cardiol. 2001;37(Suppl 1):133.

5. Dzeshka MS, Lip GYH, Snezhitskiy V, Shantsila E. Cardiac fibrosis in patients with atrial fibrillation: mechanisms and clinical implications. J Am Coll Cardiol. 2015;66(8):943-59.

6. De-Castro S, Caselli SM, Pelliccia A, Cavarretta E, Maddukuri P, Cartoni D, et al. Left ventricular remodelling index (LVRI) in various pathophysiological conditions: a real-time three-dimensional echocardiographic study. Heart. 2007; 93(2):205-9.
7. Olivotto I, Cecchi F, Casey SA, Dolara A, Traverse JH, Maron BJ. Impact of atrial fibrillation on the clinical course of hypertrophic cardiomyopathy. Circulation. 2002;11(3):58

8. Yamaji K, Fujimoto S, Yutani C, Ikeda Y, Mizuno R, Hashimoto T, et al. Does the progression of myocardial fibrosis lead to atrial fibrillation in patients with hypertrophic cardiomyopathy? Cardiovasc Pathol. 2001;10(6):297.

9. Maron BJ, Towbin JA, Thiene G, Antzelevitch C, Corrado D, Arnett D, et al. Contemporary definitions and classification of the cardiomyopathies: an American Heart Association scientific statement from the council on clinical cardiology, heart failure and transplantation committee; quality of care and outcomes research and functional genomics and translational biology interdisciplinary working groups; and council on epidemiology and prevention. Circulation. 2006;113(14):1807-16.

10. Elliott $P$, Andersson B, Arbustini E, Bilinska Z, Cecchi F, Charron P, et al. Classification of the cardiomyopathies: a position statement from the european society of cardiology working group on myocardial and pericardial diseases. Eur Heart J. 2008;29(2):270-6.

11. Schulzmenger J, Abdelaty H, Busjahn A, Wassmuth R, Pilz B, Dietz R, et al. Left ventricular outflow tract planimetry by cardiovascular magnetic resonance differentiates obstructive from non-obstructive hypertrophic cardiomyopathy. J Cardiovasc Magn Reson. 2006;8(5):741-6.

12. Schulzmenger J, Abdelaty H, Rudolph A, Elgeti T, Messroghli D, Utz W, et al. Gender-specific differences in left ventricular remodelling and fibrosis in hypertrophic cardiomyopathy: insights from cardiovascular magnetic resonance. Eur J Heart Fail. 2008;10(9):850-4.

13. Siontis KC, Geske JB, Ong K, Nishimura RA, Ommen SR, Gersh BJ. Atrial fibrillation in hypertrophic cardiomyopathy: prevalence, clinical correlations, and mortality in a large high-risk population. J Am Heart Assoc. 2014;3(3):e001002.

14. Manuguerra R, Callegari S, Corradi D. Inherited structural heart diseases with potential atrial fibrillation occurrence. J Cardiovasc Electrophysiol. 2015;27(2):242-52.

15. Losi MA, Betocchi S, Aversa M, Lombardi R, Miranda M, D'Alessandro G, et al. Determinants of atrial fibrillation development in patients with hypertrophic cardiomyopathy. Am J Cardiol. 2004;94(7):895-900.

16. Yang H, Woo A, Monakier D, Jamorski M, Fedwick K, Wigle ED, et al. Enlarged left atrial volume in hypertrophic cardiomyopathy: a marker for disease severity. J Am Soc Echocardiogr. 2005;18(10):1074-82.

17. Barbero $U$, Destefanis $P$. An Indian-look right into restrictive cardiomyopathies. Indian Heart J. 2015;67(6):512-3.

18. Poliac LC, Barron ME, Maron BJ. Hypertrophic cardiomyopathy | anesthesiology | ASA publications. Anesthesiology. 2006;104(1):183.

19. Camici PG, Olivotto I, Rimoldi OE. The coronary circulation and blood flow in left ventricular hypertrophy. J Mol Cell Cardiol. 2012;52(4):857-64.

20. Hensley N, Dietrich J, Nyhan D, Mitter N, Yee MS, Brady M. Hypertrophic cardiomyopathy: a review. Anesth Analg. 2015;120(3):554-69.

21. Barbero U, Ho SY. Anatomy of the atria: A road map to the left atrial appendage. Herzschrittmacherther Elektrophysiol. 2017;28(4):347-54.

22. Ozdemir O, Soylu M, Demir AD, Topaloglu S, Alyan O, Turhan H, et al. P-wave durations as a predictor for atrial fibrillation development in patients with hypertrophic cardiomyopathy. Int J Cardiol. 2004;94(2-3):163-6.

23. Liu T, Li G. Potential mechanisms between atrial dilatation and atrial fibrillation. Am Heart J. 2006;151(2):e1.

\section{Ready to submit your research? Choose BMC and benefit from:}

- fast, convenient online submission

- thorough peer review by experienced researchers in your field

- rapid publication on acceptance

- support for research data, including large and complex data types

- gold Open Access which fosters wider collaboration and increased citations

- maximum visibility for your research: over $100 \mathrm{M}$ website views per year

At $\mathrm{BMC}$, research is always in progress.

Learn more biomedcentral.com/submission 